Rapid Reviews COVID-19

\title{
Review 2: "Estimated transmissibility and severity of novel SARS- CoV-2 Variant of Concern 202012/01 in England"
}

Seyed Hasnain ${ }^{1}$

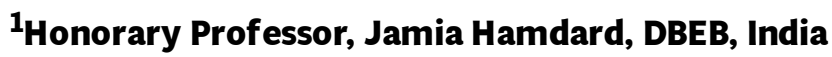

Published on: Feb 10, 2021

DOI: 10.1162/2e3983f5.06304a6e

License: Creative Commons Attribution 4.0 International License (CC-BY 4.0). 


\section{$\underline{\text { RR:C19 Evidence Scale rating by reviewer: }}$}

- Potentially informative. The main claims made are not strongly justified by the methods and data, but may yield some insight. The results and conclusions of the study may resemble those from the hypothetical ideal study, but there is substantial room for doubt. Decision-makers should consider this evidence only with a thorough understanding of its weaknesses, alongside other evidence and theory. Decision-makers should not consider this actionable, unless the weaknesses are clearly understood and there is other theory and evidence to further support it.

\section{Review:}

The current report by Nicholas et al estimates the transmissibility and impacts of the novel SARS-CoV2 Variant of Concern 202012/01 in England, also known as 501Y.V1 variant. The study design on the prediction of transmission behavior is appropriate.

The authors' concern about updating vaccination policies is justified. The represented models can also provide timely estimates to vaccine manufacturers in the wake of emerging variants.

Given the absence of data on the effects of these variants on disease severity, execution of Tier 4 or beyond measures could help reduce transmission. The authors may discuss other stringency options which might prevent further transmission.

The authors might want to discuss reliability of the models used for predictions and how their models behave compared to other models used in such analysis globally.

In conclusion, the report may provide valuable information on the spread of 501Y.V1 and other variants globally. The availability of codes such as Google mobility and Social contact data can help other researchers globally to keep check on other emerging variants.

On a scale of $1-5$, I would rate strength of evidence to be 3 . There are certain factors which could occlude exact modeling of the epidemic in England. Authors have thoroughly described these in detail in the Discussion section. Thus, the modeling predictions can be regarded as precautionary measures rather as actual data for media outlets. 